\title{
ON THE STABILITY OF THE MOTION OF A VISCOUS LIQUID*
}

\author{
BY \\ FRANCIS ROBERT SHARPE
}

§1. Introduction. ReYNolds $\dagger$ has established an energy criterion for the stability of the motion of a viscous liquid, and has applied it to the case of a liquid moving between two parallel planes. For a liquid of density $\rho$ and viscosity $\mu$, moving between two planes $2 b$ apart with mean velocity $U$ he found that the motion is unstable when

$$
\frac{2 b \rho U}{\mu}>517
$$

Using the principle that the critical velocity is inversely proportional to the hydraulic mean depth he inferred that for a cylindrical pipe of radius $a$ the critical velocity is given by

$$
\frac{2 a \rho U}{\mu}>1034,
$$

and he compared this with his experimental value 1900. In the following paper I discuss directly the case of the cylindrical pipe and find that the motion is unstable when

$$
\frac{2 a \rho U}{\mu}>470 \text {. }
$$

I also find, by using a different solution of the equation of continuity and the boundary conditions, that the motion between two parallel planes is unstable when

$$
\frac{2 b \rho U}{\mu}>167,
$$

instead of 517 as found by REYNOLDs.

§ 2. Rectilinear flow through a pipe. When the motion through a pipe is symmetrical the equations of motion, expressed in cylindrical coördinates $r, \theta, z$, are $f$

* Presented to the Society February 25, 1905. Received for publication May 5, 1905.

t Raynolds, Philosophical Transactions of the Royal Society, vol. 186 (1895) A, pp. 123-164.

$\ddagger$ LovE, Mathematical Theory of Elasticity, vol. 1, p. 217. 


$$
\begin{gathered}
\rho\left(\frac{\partial u}{\partial t}+u \frac{\partial u}{\partial r}+w \frac{\partial u}{\partial z}\right)=\frac{\partial p_{r r}}{\partial r}+\frac{\partial p_{z r}}{\partial z}+\frac{p_{r r}-p_{\theta \theta}}{r}, \\
\rho\left(\frac{\partial w}{\partial t}+u \frac{\partial w}{\partial r}+w \frac{\partial w}{\partial z}\right)=\frac{\partial p_{r z}}{\partial r}+\frac{\partial p_{z z}}{\partial z}+\frac{p_{r z}}{r}
\end{gathered}
$$

The extensions are* $\partial u / \partial r, u / r, \partial w / \partial z$. and the shear $\partial u / \partial z+\partial w / \partial r$. Hence $\dagger$

$$
\begin{gathered}
p_{r r}=-p+2 \mu \frac{\partial u}{\partial r}, \quad p_{\theta \theta}=-p+2 \mu \frac{u}{r}, \quad p_{k z}=-p+2 \mu \frac{\partial w}{\partial z}, \\
p_{\varepsilon r}=p_{r z}=\mu\left(\frac{\partial u}{\partial z}+\frac{\partial w}{\partial r}\right) .
\end{gathered}
$$

The equation of continuity is $\ddagger$

$$
\frac{\partial u}{\partial r}+\frac{u}{r}+\frac{\partial w}{\partial z}=0
$$

and the boundary conditions are

$$
u=w=0 \quad \text { when } \quad r=a .
$$

The simplest solution of these equations is $\S$

$$
\begin{gathered}
u=\frac{\partial w}{\partial t}=\frac{\partial w}{\partial z}=\frac{\partial p}{\partial r}=0, \quad \frac{\partial p}{\partial z}=\text { constant } \\
w=-\frac{1}{4 \mu} \frac{\partial p}{\partial z}\left(a^{2}-r^{2}\right)
\end{gathered}
$$

and the mean velocity of flow is

$$
U=-\frac{a^{2}}{8 \mu} \frac{\partial p}{\partial z}
$$

§3. Steady non-rectilinear motion. For some minimum value of $U$, to be determined, permanent eddying motion is possible, that is, the rectilinear motion is unstable. The mean motion is still rectilinear but $u$ is no longer zero at any particular instant. We assume then that

$$
u=u^{\prime}, \quad w=\bar{w}+w^{\prime},
$$

where $\bar{w}$ is a function of $r$, and $u^{\prime}$ and $w^{\prime}$ are periodic functions of $r$ and $z$ whose mean values are zero.

The method used by REYNOLDs is to calculate the average rate of increase of the motion $u=u^{\prime}, w=w^{\prime}$, which is the motion relative to the mean motion $u=0, w=\bar{w}$, for a volume of liquid extending to the boundaries of the liquid.

* Love, loc. cit., p. 216.

† LAMB, Hydrodynamics, p. 512, 521.

$\ddagger$ LAMB, loc. cit., p. 134.

\& LAMB, loo. cit., p. 521. 
§ 4. The equations of mean motion. Using a bar to denote a mean value we have, on substituting (5) in (1), (2) and (3),

$$
\begin{aligned}
& \rho\left\{\frac{\partial u^{\prime}}{\partial t}+u^{\prime} \frac{\partial u^{\prime}}{\partial r}+\left(\bar{w}+w^{\prime}\right) \frac{\partial u^{\prime}}{\partial z}\right\}=\frac{\partial\left(\bar{p}_{r r}+p_{r r}^{\prime}\right)}{\partial r}+\frac{\partial\left(\bar{p}_{s r}+p_{s r}^{\prime}\right)}{\partial z} \\
& +\frac{p_{r r}+p_{r_{r}}^{\prime}-\bar{p}_{\theta \theta}-p_{\theta \theta}^{\prime}}{r}, \\
& \rho\left\{\frac{\partial w^{\prime}}{\partial t}+u^{\prime} \frac{\partial w^{\prime}}{\partial r}+\left(\bar{w}+w^{\prime}\right) \frac{\partial w^{\prime}}{\partial z}+u^{\prime} \frac{\partial \bar{w}}{\partial r}\right\}=\frac{\partial\left(\bar{p}_{r z}+p_{r z}^{\prime}\right)}{\partial r} \\
& +\frac{\partial\left(\bar{p}_{s z}+p_{s s}^{\prime}\right)}{\partial z}+\frac{\bar{p}_{r z}+p_{r s}^{\prime}}{r} \\
& \frac{\partial u^{\prime}}{\partial r}+\frac{u^{\prime}}{r}+\frac{\partial w^{\prime}}{\partial z}=0 \text {. }
\end{aligned}
$$

Multiplying (6) by $\rho u^{\prime}$ and $\rho w^{\prime}$ respectively and adding to the preceding equations,

$$
\begin{aligned}
\begin{aligned}
&\left\{\frac{\partial u^{\prime}}{\partial t}+\frac{\partial\left(u^{\prime} u^{\prime}\right)}{\partial r}+\frac{\partial\left(u^{\prime} w^{\prime}\right)}{\partial z}+\bar{w} \frac{\partial u^{\prime}}{\partial z}\right.\left.+\frac{\left.u^{\prime} u^{\prime}\right\}}{r}\right\}=\frac{\partial\left(\bar{p}_{r r}+p_{r r}^{\prime}\right)}{\partial r} \\
&+\frac{\partial\left(\bar{p}_{z r}+p_{z r}^{\prime}\right)}{\partial z}+\frac{\bar{p}_{r r}+p_{r r}^{\prime}-\bar{p}_{\theta \theta}-p_{\theta \theta}^{\prime}}{r}, \\
& \rho\left\{\frac{\partial w^{\prime}}{\partial t}+\frac{\partial\left(u^{\prime} w^{\prime}\right)}{\partial r}+\frac{\partial\left(w^{\prime} w^{\prime}\right)}{\partial z}+\bar{w} \frac{\partial w^{\prime}}{\partial z}+u^{\prime} \frac{\partial \bar{w}}{\partial r}+\frac{u^{\prime} w^{\prime}}{r}\right\}=\frac{\partial\left(\bar{p}_{r r}+p_{r z}^{\prime}\right)}{\partial r} \\
&+\frac{\partial\left(p_{s z}+p_{s z}^{\prime}\right)}{\partial z}+\frac{\bar{p}_{r z}+p_{r z}^{\prime}}{r} .
\end{aligned}
\end{aligned}
$$

Taking mean values, we have the equations of mean motion

$$
\rho\left\{\frac{\partial\left(\overline{u^{\prime} u^{\prime}}\right)}{\partial r}+\frac{\overline{u^{\prime} u^{\prime}}}{r}\right\}=\frac{\partial \bar{p}_{r r}}{\partial r}+\frac{\partial \bar{p}_{s r}}{\partial z}+\frac{\bar{p}_{r r}}{r},
$$

(8) $\rho\left\{\frac{\partial\left(\overline{u^{\prime} w^{\prime}}\right)}{\partial r}+\frac{\overline{u^{\prime} w^{\prime}}}{r}\right\}=\frac{\partial \bar{p}_{r z}}{\partial r}+\frac{\partial \bar{p}_{k s}}{\partial z}+\frac{\bar{p}_{r z}}{r}=-\frac{\partial p}{\partial z}+\mu\left(\frac{\partial^{2} \bar{w}}{\partial r^{2}}+\frac{1}{r} \frac{\partial \bar{w}}{\partial r}\right)$.

Multiplying (8) by $r$ and integrating

$$
r \rho \overline{u^{\prime} w^{\prime}}=-\frac{r^{2}}{2} \frac{\partial p}{\partial z}+\mu r \frac{\partial \bar{w}}{\partial r}+C .
$$

Since this holds for $r=0$, we have $C=0$. Hence

$$
\mu \frac{\partial w}{\partial r}=\frac{r}{2} \frac{\partial p}{\partial z}+\rho \overline{u^{\prime} w^{\prime}}
$$

\$5. The energy of the relative motion. Subtracting (7) and (8) from the preceding equations we have for the relative motion, 


$$
\begin{gathered}
\rho\left\{\frac{\partial u^{\prime}}{\partial t}+\frac{\partial}{\partial r}\left(u^{\prime} u^{\prime}-\overline{u^{\prime} u^{\prime}}\right)+\bar{w} \frac{\partial u^{\prime}}{\partial z}+\frac{\partial\left(u^{\prime} w^{\prime}\right)}{\partial z}+\frac{u^{\prime} u^{\prime}-\overline{u^{\prime} u^{\prime}}}{r}\right\} \\
=\frac{\partial p_{r r}^{\prime}}{\partial r}+\frac{\partial p_{z r}^{\prime}}{\partial z}+\frac{p_{r r}^{\prime}-p_{\theta \theta}^{\prime}}{r}, \\
\begin{array}{c}
\rho\left\{\frac{\partial w^{\prime}}{\partial t}+u^{\prime} \frac{\partial w^{\prime}}{\partial r}+\frac{\partial}{\partial r}\left(u^{\prime} w^{\prime}-\overline{u^{\prime} w^{\prime}}\right)+\bar{w} \frac{\partial w^{\prime}}{\partial z}+u^{\prime} \frac{\partial \bar{w}}{\partial r}+\frac{u^{\prime} w^{\prime}-\overline{u^{\prime} w^{\prime}}}{r}\right\} \\
=\frac{\partial p_{r z}^{\prime}}{\partial r}+\frac{\partial p_{z z}^{\prime}}{\partial z}+\frac{p_{r z}^{\prime}}{r} .
\end{array}
\end{gathered}
$$

Multiplying these equations by $u^{\prime}$, and $w^{\prime}$ respectively, adding, and taking mean values,

$$
\begin{aligned}
& \left(\frac{\partial}{\partial t}+\bar{w} \frac{\partial}{\partial z}\right) \frac{1}{2} \rho\left(u^{\prime 2}+\bar{w}^{\prime 2}\right)+\rho \overline{u^{\prime} w^{\prime}} \frac{\partial \bar{w}}{\partial r}= \\
& \quad \text { mean value of } u^{\prime}\left(\frac{\partial p_{r r}^{\prime}}{\partial r}+\frac{\partial p_{z r}^{\prime}}{\partial z}+\frac{p_{r r}^{\prime}-p_{\theta \theta}^{\prime}}{r}\right)+w^{\prime}\left(\frac{\partial p_{r z}^{\prime}}{\partial r}+\frac{\partial p_{z z}^{\prime}}{\partial z}+\frac{p_{r z}^{\prime}}{r}\right) .
\end{aligned}
$$

Integrating over the section of the pipe and by parts on the right hand side, dividing by $2 \pi$, and remembering that $u^{\prime}, w^{\prime}$ vanish on the boundary, we obtain the equation

$$
\begin{gathered}
\int_{0}^{a}\left(\frac{\partial}{\partial t}+\bar{w} \frac{\partial}{\partial z}\right) \frac{1}{2} \rho\left(\bar{u}^{\prime 2}+\bar{w}^{\prime 2}\right) \cdot r d r=\int_{0}^{a}-\rho \overline{u^{\prime} w^{\prime}} \frac{\partial \bar{v}}{\partial r} \cdot r d r \\
-\mu \int_{0}^{a}\left[2\left(\frac{\partial u^{\prime}}{\partial r}\right)^{2}+2\left(\begin{array}{c}
\partial w^{\prime} \\
\partial z
\end{array}\right)^{2}+2 \frac{u^{\prime 2}}{r^{2}}+\left(\frac{\partial u^{\prime}}{\partial z}+\frac{\partial w^{\prime}}{\partial r}\right)^{2}\right] r d r,
\end{gathered}
$$

where, in the second integral on the right, mean values are to be taken.

The left hand side of this equation is the average rate of increase of the energy of the relative motion of a length $1 / 2 \pi$ of liquid in the pipe.

Hence we have REYNOLD's criterion for the possibility of permanent eddying motion expressed in cylindrical coördinates, namely,

$$
\begin{aligned}
-\int_{0}^{a} \rho \overline{u^{\prime} w^{\prime}} \frac{\partial \bar{w}}{\partial r} \cdot r d r \geqq \mu \int_{0}^{a}[ & 2\left(\frac{\partial u^{\prime}}{\partial r}\right)^{2}+2\left(\frac{\partial w^{\prime}}{\partial z}\right)^{2} \\
& \left.+2 \frac{u^{\prime 2}}{r^{2}}+\left(\frac{\partial u^{\prime}}{\partial z}+\frac{\partial w^{\prime}}{\partial r}\right)^{2}\right] r d r
\end{aligned}
$$

where, in the integrand on the right, mean values are to be taken.

The interpretation of equations (9) and (10) is that in order to maintain the relative as well as the mean motion an increased pressure gradient is necessary and the additional energy is dissipated in the relative motion, the right-hand 
integrand of (10) being STokEs's dissipation function expressed in cylindrical coördinates.

§6. Determination of a possible relative motion. The solution of the continuity equation (6) with the boundary conditions $u^{\prime}=w^{\prime}=0$, for $r=a$, which leads to a minimum value of $U$ is

$$
\begin{gathered}
u^{\prime}=L A \frac{\pi r}{2 a}(\sin p+\sin 3 p) \sin \frac{\pi L z}{2 a}-L B \frac{\pi r}{2 a}\left(\sin 2 p+\frac{1}{2} \sin 4 p\right) \cos \frac{\pi L z}{2 a} \\
w^{\prime}=A\left\{2(\sin p+\sin 3 p)+\frac{\pi r}{2 a}(\cos p+3 \cos 3 p)\right\} \cos \frac{\pi L z}{2 a} \\
+B\left\{2\left(\sin 2 p+\frac{1}{2} \sin 4 p\right)+\frac{\pi r}{2 a}(2 \cos 2 p+2 \cos 4 p)\right\} \sin \frac{\pi L z}{2 a},
\end{gathered}
$$

as is easily seen on substitution, $p$ being $\pi r / 2 a$.

These give

$$
\overline{u^{\prime} w^{\prime}}=\frac{L A B \pi^{2} r^{2}}{32 a^{2}}(3 \sin p+3 \sin 3 p-\sin 5 p-\sin 7 p) .
$$

Hence from (9) we find, neglecting $\overline{u^{\prime} w^{\prime 2}}$, the left-hand side of equation (10) equal to

$-\frac{L A B \pi^{2} \rho U}{64 a^{2} \mu} \int_{0}^{a} r^{4}(3 \sin p+3 \sin 3 p-\sin 5 p-\sin 7 p) d r=.215 L A B a \rho U$.

Substituting the values of $u^{\prime}$ and $w^{\prime}$ in the right hand side of $(10)$, taking mean values, and integrating, we obtain the condition

$$
\begin{aligned}
\frac{.215 L A B a \rho U}{\mu} \geqq( & \left..304 A^{2}+.127 B^{2}\right) L^{4} \\
& +\left(4.26 A^{2}+6.67 B^{2}\right) L^{2}+35.7 A^{2}+16.34 B^{2} .
\end{aligned}
$$

Hence for a minimum value of $U$ we have to minimize

$$
\left(\begin{array}{c}
.304 \\
\lambda
\end{array}+.127 \lambda\right) L^{3}+\left(\frac{4.26}{\lambda}+6.67 \lambda\right) L+\left(\frac{35.7}{\lambda}+16.34 \lambda\right) \frac{1}{L}
$$

where $\lambda=B / A$.

Differentiating with respect to $L$ and $\lambda$ we find

$$
\begin{aligned}
3\left(.304+.127 \lambda^{2}\right) L^{4}+\left(4.26+6.67 \lambda^{2}\right) L^{2}-35.7-16.34 \lambda^{2}=0, \\
\left(-.304+.127 \lambda^{2}\right) L^{4}+\left(-4.26+6.67 \lambda^{2}\right) L^{2}-35.7+16.34 \lambda^{2}=0 .
\end{aligned}
$$

Eliminating $\lambda^{2}$,

$$
.4633 L^{8}+20.55 L^{6}+151.67 L^{4}-2333.35=0 \text {. }
$$


Hence $L=1.798, \lambda=1.158$, approximately. The minimum value of $U$ is therefore given by

REYNolds' experiments gave

$$
\frac{2 a \rho U}{\mu}=470 \text {. }
$$

$$
\frac{2 a \rho U}{\mu}=1900 \text {. }
$$

REYNOLDs' result for the flow between two parallel planes a distance $2 b$ apart is

$$
\frac{2 b \rho U}{\mu}=517 \text {, }
$$

and because the hydraulic mean depth for a cylinder radius $a$ is $\frac{1}{2} a$ and for two parallel planes is $b$ he inferred that for a cylinder

$$
\frac{2 a \rho U}{\mu}=1034 \text {. }
$$

§ 7. Flow between parallel planes. When the motion is the same in planes parallel to the $x y$ plane the equations of motion are *

where

$$
\begin{aligned}
& \rho\left(\frac{\partial u}{\partial t}+u \frac{\partial u}{\partial x}+v \frac{\partial u}{\partial y}\right)=\frac{\partial p_{x x}}{\partial x}+\frac{\partial p_{y x}}{\partial y} \\
& \rho\left(\frac{\partial v}{\partial t}+u \frac{\partial v}{\partial x}+v \frac{\partial v}{\partial y}\right)=\frac{\partial p_{x y}}{\partial x}+\frac{\partial p_{y y}}{\partial y}
\end{aligned}
$$

$$
\begin{gathered}
p_{x x}=-p+2 \mu \frac{\partial u}{\partial x}, p_{y y}=-p+2 \mu \frac{\partial u}{\partial y}, \\
p_{y x}=p_{x y}=\mu\left(\begin{array}{l}
\partial u \\
\partial y
\end{array}+\frac{\partial v}{\partial x}\right) .
\end{gathered}
$$

The equation of continuity is

$$
\frac{\partial u}{\partial x}+\frac{\partial v}{\partial y}=0
$$

In the case of liquid flowing between two parallel planes $y= \pm b$ the boundary conditions are $u=v=0$ when $y= \pm b$.

The simplest solution of these equations is

$$
v=\begin{aligned}
& \partial u \\
& \partial x
\end{aligned}=\frac{\partial p}{\partial y}=0, \quad \frac{\partial p}{\partial x}=\text { constant }, \quad u=\frac{1}{2 \mu} \frac{\partial p}{\partial x}\left(y^{2}-b^{2}\right),
$$

and the mean velocity of flow is

$$
U=-\begin{gathered}
b^{2} \\
\partial p \\
3 \mu \\
\partial x
\end{gathered}
$$

* LAM1, Hydrodynamics, pp. 512-520. 
Using the method of the preceding problem we find for the equation of mean motion

and the criterion of stability,

$$
\rho \frac{\partial\left(u^{\prime} v^{\prime}\right)}{\partial y}=-\frac{\partial p}{\partial x}+\mu \frac{\partial^{2} \bar{u}}{\partial y^{2}}
$$

$$
-\int_{-b}^{b} \rho \overline{u^{\prime} v^{\prime}} \frac{\partial \bar{u}}{\partial y} d y \geqq \mu \int_{-b}^{+b}\left\{2\left(\frac{\partial u^{\prime}}{\partial x}\right)^{2}+2\left(\frac{\partial v^{\prime}}{\partial y}\right)^{2}+\left(\frac{\partial u^{\prime}}{\partial y}+\frac{\partial v^{\prime}}{\partial x}\right)^{2}\right\} d y .
$$

\$ 8. Determination of a possible relative motion. The solution of the continuity equation, with the boundary conditions, which leads to a minimum value of $U$ is

$$
\begin{aligned}
& u^{\prime}=A(\sin p+\sin 3 p) \cos \frac{\pi L x}{2 b}+B(2 \sin 2 p+4 \sin 4 p) \sin \frac{\pi L x}{2 b}, \\
& v^{\prime}=-L A\left(\cos p+\frac{1}{3} \cos 3 p\right) \sin \frac{\pi L x}{2} b+L B(\cos 2 p+\cos 4 p) \cos \frac{\pi L x}{2 b},
\end{aligned}
$$

These give

$$
4 \overline{u^{\prime} v^{\prime}}=-L A B\left\{\frac{11}{3} \sin p+6 \sin 3 p+\frac{8}{3} \sin 5 p+\frac{1}{3} \sin 7 p\right\} .
$$

To determine $\partial \bar{u}, \partial y$ we have, on integrating (11),

$$
\overline{\rho u^{\prime} v^{\prime}}=-y \frac{\partial p}{\partial x}+\mu \frac{\partial \bar{u}}{\partial y}+C .
$$

Now $\overline{u^{\prime} v^{\prime}}$ is an odd function of $y$ and so is $\partial \bar{u} / \partial y$ since the mean motion is symmetrical with respect to the planes $y= \pm b$. Hence $C$ is zero and approxi- . mately

$$
\frac{\partial \bar{u}}{\partial y}=\frac{y}{\mu} \frac{\partial p}{\partial x}=-\frac{3 U y}{b^{2}}
$$

Hence the left hand side of $(12)$ reduces to

$$
\frac{-18.6 L A B \rho U}{\pi^{2}} \text {. }
$$

Substituting the values of $u^{\prime}$ and $v^{\prime}$ in the right hand side of (12), taking mean values, and integrating, we have the condition

$$
\begin{aligned}
& \frac{-18.6 L A B \rho U}{\pi^{2}} \\
& \geqq \frac{\mu b}{2}\left\{\frac{\pi^{2} L^{4}}{4 b^{2}}\left(\frac{10}{9}-A^{2}+2 B^{2}\right)+\frac{2 \pi^{2} L^{2}}{4 b^{2}}\left(2 A^{2}+20 B^{2}\right)+\frac{\pi^{2}}{4 b^{2}}\left(10 A^{2}+272 B^{2}\right)\right\}, \\
& \frac{3 b \rho U}{\mu} \geqq\left(\begin{array}{c}
\pi \\
2
\end{array}\right) \frac{\left(\frac{10}{9} A^{2}+2 B^{2}\right) L^{4}+\left(4 A^{2}+40 B^{2}\right) L^{2}+10 A^{2}+272 B^{2}}{-3.1 L A B} .
\end{aligned}
$$


REYNOLDS takes the solution

$$
\begin{aligned}
& u^{\prime}=A(\cos p+3 \cos 3 p) \cos \frac{\pi L x}{2 b}+B(2 \cos 2 p+2 \cos 4 p) \sin \frac{\pi L x}{2 b^{-}}, \\
& v^{\prime}=L A(\sin p+\sin 3 p) \sin \frac{\pi L x}{2 b}-L B\left(\sin 2 p+\frac{1}{2} \sin 4 p\right) \cos \frac{\pi L x}{2 b^{-}},
\end{aligned}
$$

and obtains the condition

$$
\frac{3 b U \rho}{\mu} \geqq\left(\frac{\pi}{\overline{2}}\right)^{4} \frac{\left(2 A^{2}+\frac{5}{4} B^{2}\right) L^{4}+\left(20 A^{2}+16 B^{2}\right) L^{2}+82 A^{2}+80 B^{2}}{-1.325 L A B} .
$$

The coefficients of $A^{2}$ and $B^{2}$ being nearly equal, he determines the minimum value of $U$ by assuming $A=B$.

It is interesting to notice that his value for $v^{\prime}$ vanishes midway between the planes and $u^{\prime}$ is there a maximum whereas the values here adopted give a maximurn value of $v^{\prime}$ midway betweer the planes, which seem to be more in accordance with experiments.

Using the method of the preceding problem we find a minimum value of $U$ given by

$$
\frac{3 b U \rho}{\mu}=41\left(\frac{\pi}{2}\right)^{4}
$$

when $L=1.398$ and $A / B=4.03$.

Hence our final result is that the motion is unstable when

REYNoLDs' result is 517 .

$$
\frac{2 b U \rho}{\mu} \geqq 167 \text {. }
$$

Cornell University, April, 1905. 